Meta

Journal des traducteurs

Translators' Journal

\title{
L'enseignement de la traduction littéraire dans le cadre d'un baccalauréat spécialisé en traduction
}

\section{Christel Gallant}

Volume 33, numéro 2, juin 1988

L'enseignement de la traduction au Canada - Teaching Translation

in Canada

URI : https://id.erudit.org/iderudit/002789ar

DOI : https://doi.org/10.7202/002789ar

Aller au sommaire du numéro

Éditeur(s)

Les Presses de l'Université de Montréal

ISSN

0026-0452 (imprimé)

1492-1421 (numérique)

Découvrir la revue

Citer cet article

Gallant, C. (1988). L'enseignement de la traduction littéraire dans le cadre d'un baccalauréat spécialisé en traduction. Meta, 33(2), 216-226.

https://doi.org/10.7202/002789ar d'utilisation que vous pouvez consulter en ligne. 


\title{
L'ENSEIGNEMENT DE LA TRADUCTION LITTÉRAIRE DANS LE CADRE D'UN BACCALAURÉAT SPÉCIALISÉ EN TRADUCTION
}

\author{
Christel GallaNT \\ Université de Moncton, Moncton, Nouveau-Brunswick
}

\section{INTRODUCTION}

Les cours de traduction littéraire, version et thème, de rigueur dans le cadre de ma propre formation de traductrice, consistaient en la traduction commentée de pages choisies d'auteurs célèbres comme André Gide ou Thomas Mann. Je les ai suivis avec beaucoup d'intérêt, beaucoup de conviction et, somme toute, beaucoup de profit. Ce n'est que plus tard, après avoir étudié davantage les théories de la traduction et la littérature, que je me suis rendu compte de leur totale inutilité pour la compréhension de ce qui fait la particularité de la traduction littéraire.

Il n'est donc pas étonnant que j'aie préféré explorer une autre approche quand, il y a huit ans, je me vis confier la responsabilité d'un cours de traduction littéraire offert comme cours à option aux étudiants des deux dernières années de notre baccalauréat spécialisé en traduction'. Sans avoir la prétention de proposer une méthode, je voudrais rendre compte d'une orientation qui, affinée au fil des ans, s'est avérée, à mon avis, fructueuse et efficace. Comme il me parait probable que d'autres appliquent des modèles analogues dans leurs cours, j'espère que cette description servira d'amorce à des échanges entre professeurs dans un domaine où les articles sur la pédagogie sont encore plus rares que ceux qui portent sur l'enseignement de la traduction pragmatique.

Après avoir passé en revue les principaux obstacles auxquels se heurte l'enseignement de la traduction littéraire dans un programme de premier cycle et proposé quelques buts à atteindre, je tâcherai d'esquisser tour à tour la philosophie de mon cours, son déroulement et les résultats observés.

\section{LA PLACE DE LA TRADUCTION LITTÉRAIRE DANS LES PROGRAMMES DE TRADUCTION}

La traduction littéraire occupe souvent une place contestée et mal définie dans les programmes de traduction, et ceci pour plusieurs raisons. D'un côté, et peut-être surtout en Europe, elle reste suspecte aux yeux des praticiens de la traduction technique, qui, historiquement, ont eu quelques difficultés à établir leur image de marque face à celle du traducteur littéraire, considéré pendant longtemps comme le seul représentant véritable de la traduction. D'un autre côté, les cours de traduction littéraire inspirent toujours une certaine méfiance dans un programme de traduction où l'on favorise en général un style d'enseignement qui prépare les aspirants traducteurs aussi rigoureusement que possible à leur future vie professionnelle.

A ce climat peu accueillant s'ajoutent les réticences des traducteurs littéraires eux-mêmes. Beaucoup parmi eux ont dit, ou laissé entendre, que la traduction littéraire est un art qui ne s'enseigne pas, que l'on devient traducteur littéraire comme on devient écrivain, par goût et par nécessité intérieure plutôt que par formation, et que, par consé- 
quent, le métier de traducteur littéraire, comme celui d'écrivain, repose avant tout sur un apprentissage solitaire, dépendant de la personnalité.

D'ailleurs, parmi les professeurs, rares sont ceux - s'il y en a - qui ont la prétention de former de futurs traducteurs littéraires, soit parce que l'expérience leur a montré que leurs étudiants ne présentent que très exceptionnellement les aptitudes nécessaires, soit parce qu'ils reconnaissent que la traduction littéraire, n'étant pas simplement une forme de traduction spécialisée au même titre que la traduction économique ou juridique par exemple, pose des problèmes particuliers de structuration de l'enseignement. Car, s'il est vrai que pour l'apprentissage de la traduction de textes spécialisés il n'y a pas de solutions passe-partout, il n'en reste pas moins que, contrairement aux écrivains, les ingénieurs et les juristes ont reçu une formation spécialisée, et qu'il est possible de puiser dans celle-ci pour dégager un bagage cognitif de base à l'usage des futurs traducteurs. La matière des cuvres littéraires, par contre, reste par définition individuelle, souvent unique, imprévisible. Il en va de même du style de l'écrivain, alors qu'il existe dans chaque langue un style technique, administratif, économique ou juridique accepté ou consacré permettant l'acquisition et la réutilisation d'un fonds de phraséologie typique.

Malgré toutes ces réticences et ces incertitudes, la traduction littéraire est perçue comme un phénomène culturel trop important pour être laissée pour compte et continue donc à faire l'objet de cours. Au premier cycle, ces cours consistaient traditionnellement en la traduction de pages choisies, parfois pour illustrer " l'intraduisibilité ", mais le plus souvent pour permettre aux étudiants de pratiquer un niveau de langue qu'ils rencontrent rarement dans leurs autres cours. Il ne fait aucun doute que de tels cours peuvent être utiles dans l'acquisition d'une plus grande maîtrise des langues de travail du traducteur. Mais puisqu'il est difficile de montrer, dans le cadre d'un paragraphe ou d'un extrait de texte, en quoi les problèmes de la traduction littéraire diffèrent de ceux de la traduction pragmatique, une telle orientation présente toujours le danger de ne pas dépasser le microcontexte et d'ancrer davantage la conception, déjà trop répandue, que la littérature est uniquement une question de style, d'images et d'esthétique.

Plus récemment, et sans doute en partie à cause de ces difficultés, l'on observe une tendance à réserver l'étude de la traduction littéraire aux programmes de maîtrise. On fait alors une plus grande place à l'analyse de traductions existantes, que l'on examine à la lumière des théories de la traduction, des différents postulats de la linguistique, ou des systèmes socioculturels dont l'original et sa traduction sont tributaires. Plus adéquate pour rendre compte de la spécificité de la traduction littéraire, cette approche n'est cependant pas non plus tout à fait exempte de pièges, dont le principal me semble résider dans la tentation, toujours présente, de porter toute l'attention sur les divergences et les difficultés, particulièrement révélatrices aux yeux du linguiste, et de glisser trop rapidement sur l'examen de la traduction réussie, quantitativement bien plus importante.

Mais quelle que soit l'orientation que l'on entend donner aux cours de maitrise, si l'on ne veut pas voir se généraliser les préjugés selon lesquels la traduction littéraire n'est qu'un mode de traduction marginal, démodé et difficile, ou, au contraire, un exercice analogue à la traduction spécialisée mais avec le style en plus, il est important de donner aux étudiants la possibilité de l'examiner de plus près dès le premier cycle, qui pour beaucoup restera le seul.

Plutôt que d'être conçu comme un élément de perfectionnement linguistique et donc un complément de la formation professionnelle, un cours de traduction littéraire au premier cycle devrait viser une certaine sensibilisation des futurs traducteurs au travail de leurs collègues littéraires, dont ils dépendent d'ailleurs eux aussi dès qu'ils lisent des auteurs qui n'appartiennent ni au monde anglo-saxon $\mathrm{ni}$ au monde francophone. 
Le but d'un tel cours n'étant ni d'apprendre à traduire des textes littéraires ni de théoriser sur la traduction, il convient de choisir un style d'enseignement fondé sur l'exploration et de viser l'apprentissage non pas d'un savoir-faire, mais d'un savoircomprendre. C'est en fonction de ces considérations que j'ai tenté d'orienter mon approche pédagogique.

\section{L'ORIENTATION DU COURS}

L'orientation générale du cours part du principe que, dans son essence, la traduction littéraire s'inscrit parfaitement dans la théorie du sens, professée par l'École de Paris, et dont le nom initial " théorie interprétative de la traduction ${ }^{2}$ ", moins frappant, mais aussi moins restrictif, convient en fait mieux à la traduction d'une œuvre de création. On sait que selon cette théorie, la traduction, loin d'être une opération purement linguistique, repose en fait sur la reproduction d'un processus mental déclenché et guidé par la force motrice du vouloir-dire qui aboutit à l'expression de celui-ci par le moyen de la langue, parlée ou écrite. Au lieu d'être mû par sa propre pensée, par son propre message, ou par son propre vécu, l'interprète, le traducteur ou le traducteur littéraire part de la pensée, du message ou du vécu d'un autre. Ce vouloir-dire, il l'écoute, le lit ou s'y expose, pour le faire sien et le dire, le rédiger ou le recréer à son tour. C'est donc le même processus fondamental qui, dans le cas de l'interprète, aboutit à un acte de parole, dans le cas du traducteur, à un acte de rédaction et dans le cas d'un traducteur littéraire, à un acte de création. Dans chaque cas, l'intermédiaire agit comme s'il était lui-même l'orateur, l'auteur ou l'écrivain et dans chaque cas les facultés intellectuelles ou émotives qui entrent en jeu sont du même type que celles qui ont abouti à l'existence de l'original. Ainsi, la traduction idéale correspond à la compréhension et l'assimilation parfaites du vouloir-dire original, doublées d'une parfaite équivalence des opérations d'expression.

Cela implique que certaines imperfections ou distorsions, qu'on attribue parfois trop vite à des impossibilités de traduction dues à des incompatibilités entre les langues en cause, résultent en réalité du fait que ces conditions préalables ne sont pas pleinement atteintes. Dans le cas de la traduction littéraire par exemple, tel traducteur n'a peut-être pas l'envergure de l'auteur qu'il traduit, sa personnalité et sa sensibilité sont trop différentes de celles de l'auteur, ou encore les conditions de travail qu'on lui impose l'obligent soit à abréger le processus d'interprétation et à reproduire une œuvre qu'il n'a que très imparfaitement "fait sienne", soit à escamoter le processus de vérification assurant que la traduction correspond effectivement à l'original sans en rétrécir ni étendre les possibilités d'interprétation.

Dans son schéma du processus heuristique de la traduction ${ }^{3}$, Delisle indique en effet comment le bon traducteur passe dans son travail par une phase distincte de deuxième interprétation ou réinterprétation lui permettant des retouches - tout comme l'auteur d'un texte écrit a la possibilité de reprendre son texte si, à la relecture, il ne reflète pas pleinement son vouloir-dire ${ }^{11}$.

La traduction littéraire comporte bien entendu aussi cette obligation d'une rétrointerprétation justificative, qui ne s'exerce pas seulement sur des questions de détail, mais porte en même temps sur l'œuvre dans son entité, comme les traducteurs littéraires le répètent depuis longtemps. L'œuvre littéraire ne se réfère pas à une réalité extérieure vérifiable, mais crée sa propre réalité qui doit être respectée. Il en résulte une multiplicité de paramètres internes qui rendent l'interprétation plus complexe que dans le cas d'un texte pragmatique. Or, dans un cours de traduction littéraire où l'on se limite aux exercices de traduction portant sur des pages choisies, cet aspect est largement négligé, ou du moins il n'entre pas dans l'expérience de l'étudiant. Traduire un passage d'un roman sans une connaissance intime de l'ceuvre dans son ensemble équivaut pourtant, en traduction littéraire, à travailler sur une phrase détachée de son contexte en tra- 
duction pragmatique et, pour reprendre une distinction chère à l'École de Paris, à traduire la langue au lieu de traduire l'œuvre.

Convaincue que la réceptivité, la faculté du faire-sien, est, tout autant que la faculté d'expression, une condition sine qua non du bon traducteur, je choisis donc de prendre résolument le contrepied des cours que j'ai moi-même suivis. Il s'agit pour moi de faire parcourir à l'étudiant les deux phases qui entourent et accompagnent l'acte de reformulation. Au lieu de lui demander de traduire, je lui demande une double lecture : celle de l'œuvre comme s'il devait la traduire et celle de la traduction d'un autre comme s'il l'avait écrite lui-même et devait pouvoir la justifier. Sans englober l'ensemble du processus de la traduction littéraire, cette approche, qui en privilégie l'étape initiale et l'étape finale, me paraît au moins aussi légitime, et infiniment plus prometteuse au niveau du płemier cycle, qu'une méthode entièrement centrée sur le moment de l'écriture.

Il faut toutefois prendre quelques précautions pour respecter le caractère dynamique du processus de traduction et ne pas verser dans la comparaison de textes pure et simple. Il convient ainsi de séparer nettement les deux phases d'interprétation. La première interprétation se fait sans connaissance préalable de la traduction. Sans être intégrale, elle permettra de cerner un certain nombre d'éléments importants ou caractéristiques de l'ouvre. La lecture de la traduction, comme un tout en soi, se fera d'abord sans recours à l'original. Ce n'est qu'après avoir comparé les deux expériences de lecture, qu'on tentera, lors de la deuxième interprétation, de déterminer le degré d'équivalence en analysant notamment les aspects identifiés auparavant comme éléments caractéristiques de l'original.

Pour un cours au niveau du premier cycle, l'approche choisie présente quatre avantages importants. Premièrement, elle permet de prendre appui sur les ressources passives et réceptives des étudiants, normalement plus étendues que leurs aptitudes actives et créatrices. L'obligation de faire porter l'analyse justificative sur le texte d'un autre leur évite en général les problèmes de cercle vicieux, tout en élargissant le champ de vision, et élimine l'effet perturbateur des mécanismes de défense dans l'examen de la compatibilité avec l'original. Deuxièmement, en faisant appel à la lecture plutôt qu'à l'écriture, elle permet de respecter l'exigence de l'entité de l'œuvre tout en basant l'essentiel du cours sur des genres littéraires longs, mais qui conviennent particulièrement bien à une initiation : le roman, la nouvelle, le récit et le conte, éventuellement même une pièce de théâtre. Troisièmement, elle permet de s'inspirer des méthodes d'une discipline qui étudie justement les multiples aspects qui interviennent dans la lecture attentive et la compréhension d'une œuvre : la recherche littéraire. Ceci se justifie d'autant plus que les étudiants ont déjà été exposés à la théorie et à la pratique du processus de traduction en général, et qu'il est donc possible de compter sur un certain acquis dans ce domaine, alors que leurs contacts le plus souvent sporadiques avec la littérature nécessitent un peu de rattrapage. Quatrièmement, elle permet de guider l'étudiant vers un travail individuel d'envergure, susceptible de respecter ses affinités personnelles, et dont le compte rendu contribuera à son tour à assurer au groupe une certaine variété des perspectives.

Toute la structure du cours est conçue en fonction de ce travail auquel il faut préparer l'étudiant, l'objectif étant de développer chez lui une démarche cadre pour l'assimilation d'une cuvre importante et l'analyse de sa traduction, non pas selon une échelle de valeurs donnée, mais à partir du bagage de connaissances et de sensibilité qu'il possède au départ.

Le style pédagogique relève de la méthode inductive et se fonde sur une exploration compatible avec les capacités moyennes d'un étudiant de premier cycle. 


\section{LE DÉROULEMENT DU COURS}

Le cours se divise en trois étapes. La première constitue une phase de prise de conscience destinée à permettre aux étudiants de réagir à certains aspects de la traduction littéraire auxquels le professeur les expose, et au professeur de mieux cerner les attentes et les capacités individuelles des étudiants afin de choisir en conséquence parmi différentes possibilités de textes pour l'étape suivante. La deuxième étape consiste en des exercices plus systématiques de lecture interprétative d'une cuvre, en vue de sa traduction, comportant en général une courte expérience de traduction active, et en l'étude de certaines prises de position des traducteurs littéraires eux-mêmes ${ }^{4}$. Elle vise aussi la mise au point d'une grille de présentation pour les exposés finals. Pendant les deux premières parties du cours, le travail se fait en commun, et le style exploratoire choisi permet à l'étudiant de toucher brièvement à certains aspects de la traduction littéraire qu'il ne rencontrera probablement pas dans son travail, comme la traduction poétique, à laquelle se résume à tort la traduction littéraire dans l'esprit de beaucoup de gens, ou la traduction pour le théâtre. La troisième étape est individuelle : à la lumière de l'expérience acquise dans les deux premières parties du cours, l'étudiant se charge de la description interprétative d'un roman et de sa traduction. Elle comporte une partie d'encadrement par le professeur, qui conseille chaque étudiant en fonction de l'ouvre choisie, et une partie de mise en commun, où les étudiants, tour à tour, rendent compte des interprétations dont ils ont assumé la responsabilité.

Afin d'illustrer quelque peu le déroulement du cours dans la pratique, j'évoquerai brièvement quelques éléments typiques de chacune des phases en indiquant sommairement les principales options de discussion qu'ils offrent.

La phase de prise de conscience ayant pour but d'éveiller l'attention et de stimuler la réflexion des étudiants comporte en général l'examen d'un certain nombre d'exemples frappants, qui peuvent fort bien être disparates ou isolés, à condition de pouvoir servir de tremplin pour des discussions et des compléments d'information sur un ou plusieurs problèmes particuliers.

Ainsi j'amorce parfois les échanges par la distribution d'un extrait de quelques lignes de Comme il vous plaira, en le situant dans la pièce : le fou se moque des effusions poétiques du jeune amoureux dans lesquelles le nom de la bien-aimée, Rosalind, revient un peu trop souvent à son goût, et il prétend pouvoir en faire autant sans le moindre effort.

Touchstone : For a taste;

If a hart do lack a hind,

Let him seek out Rosalind.

If the cat will after kind,

So be sure will Rosalind.

Winter garments must be lin'd,

So must slender Rosalind.

They that reap must sheaf and bind;

Then to cart with Rosalind.

Sweetest nut hath sourest rind,

Such a nut is Rosalind.

He that sweetest rose will find,

Must find love's prick and Rosalind.

This is the very false gallop of verses: why do you infect yourself with them ${ }^{5}$ ? 
L'intérêt suscité n'est peut-être pas très grand, mais on fait preuve de bonne volonté. Après avoir mentionné au passage l'importance de Shakespeare pour les Romantiques, j'invite ensuite les étudiants à lire la traduction de François-Victor Hugo.

PIERRE DE TOUCHE. - Un léger essai :

Si un cerf veut une biche.

Qu'il aille trouver Rosalinde!

Si la chatte court après son mâle

Ainsi certes fera Rosalinde.

Habit d'hiver doit être doublé,

Et de même la mince Rosalinde.

Pour moissonner, il faut gerber et lier,

Puis charrier avec Rosalinde.

La plus douce noix a la plus aigre écorce :

Cette noix, c'est Rosalinde.

Qui veut trouver la plus suave rose,

Trouve épine d'amour et Rosalinde!

C'est là le faux galop du vers : pourquoi vous empestez-vous de pareilles rimes ${ }^{6}$ ?

Cette fois, on observe des réactions marquées d'indifférence jusqu'à la distribution de la traduction de Jean Anouilh qui provoque des remous.

Qui peut donc fleurer le benjoin de

La façon de Rosalinde?

La seule orange sans pépin de

L'univers, est Rosalinde?

Toute musique est un crincrin depuis que j'entends Rosalinde!

Il n'est plus de grâce au jasmin devant le teint de Rosalinde...

Un nectar, l'huile de ricin, de

La main de Rosalinde!

Le monde est pour moi un écrin de

Ce bijou qu'est Rosalinde...

Rien n'est plus aisé qu'un refrain de

La chanson de Rosalinde,

Il vous suffit d'avoir un grain de

Folie... et la rime en inde !... reilles $^{7}$ ?

Voilà le truc, c'est tout simple! Pourquoi vous empoisonner avec des choses pa-

Les réactions sont en général très vives et peuvent être canalisées par des questions comme "Qu'a voulu faire chacun des traducteurs ? "Quelles sont les conséquences de son choix pour l'usager de traduction ? " et la question classique : "Fidélité à quoi ? " L'exemple mène généralement à une discussion intéressante et utile sur les particularités de la traduction théâtrale, que l'on peut étoffer par la lecture commentée de quelques articles écrits par les traducteurs littéraires à ce sujet, ou approfondir, soit par la comparaison des traductions d'Anouilh et de F.-V. Hugo de toute une scène de Comme il vous plaira - avec possibilité d'enchaînement sur le problème de la modernisation des traductions -, soit par l'examen du traitement des chansons par les deux traducteurs - 
avec possibilité d'enchaînement sur la traduction poétique. Il est également possible de soulever ici le problème du rôle du traducteur dans la réception d'un auteur, et, s'il y a des étudiants germanisants dans la classe, de spéculer brièvement sur les raisons de la place très différente qu'occupent les pièces de Shakespeare dans les répertoires des théâtres français et allemands. Quelle que soit la voie choisie, il ne s'agit pas d'aboutir à une position normative ou à un modèle théorique, mais d'amener les étudiants à prendre conscience de la multiplicité des facettes de la traduction littéraire.

Si l'on veut lancer une discussion analogue sur la traduction poétique, et qu'on a devant soi un groupe d'étudiants qui affiche une certaine timidité devant la poésie, comme cela arrive assez souvent, il peut être pédagogiquement efficace de prendre comme point de départ la traduction vers la deuxième langue d'un poème en langue maternelle et susceptible de toucher la plupart d'entre eux. Ainsi, pour mes étudiants, en majorité acadiens, je choisis volontiers un des de poèmes publiés dans Ellipse ${ }^{8}$, dont ils connaissent d'ailleurs souvent l'auteur et les traducteurs, car le réflexe d'appartenance et l'identification psychologique compensent souvent des faiblesses du "sens poétique".

Là encore, la lecture et la discussion d'articles pertinents permettra d'élargir ensuite le champ de vision et de se rendre compte, par exemple, qu'il existe des moyens d'analyse bien plus rigoureux que l'approche subjective suivie jusqu'ici.

Finalement, il me paraît éclairant de faire l'expérience d'une traduction à partir d'une langue accessible, c'est-à-dire écrite en lettres latines, mais inconnue, comme l'italien, le néerlandais, le polonais ou une langue nordique. La lecture interprétative commence cette fois par la traduction, car pour mesurer les services que rend le traducteur littéraire à un lecteur cantonné dans sa langue maternelle ou un nombre de langues très limité, il peut être révélateur de lire et d'analyser une traduction et de se tourner ensuite vers un original dont la "lecture" n'apporte rien de plus!

C'est un moment propice pour rappeler que la littérature dite universelle ne l'est que grâce aux traductions, qui font entrer les héros des romans dans le patrimoine commun. On peut aussi chercher ensemble des exemples d'œuvres "portées à l'écran " à partir non pas de l'original, mais d'une traduction, ou présenter aux étudiants une collection d'échantillons de critique littéraire dont les auteurs assument de toute évidence une identité œuvre-traduction.

Dans la deuxième phase du cours, consacrée à la lecture interprétative d'œuvres en prose et à la justification de leur traduction, ainsi qu'à la traduction active d'une œuvre très courte, les exercices sont plus systématiques sans être pour autant très ambitieux. Ainsi, l'exercice de double interprétation peut être thématique et se limiter à un ou deux aspects : tout ce que l'on sait des personnages par exemple, de leur caractère et de leur physique, tout ce que l'on peut déduire et pourquoi, ce que le texte ne dit pas et que l'on voudrait savoir (ou que le lecteur complète spontanément), ce que le texte ne dit pas et qu'on n'éprouve pas le besoin d'imaginer. On analyse la traduction de la même façon pour différencier entre la démarche d'un lecteur normal (ou encore d'un metteur en scène, d'un réalisateur de film), qui peut se permettre une interprétation très subjective, et celle d'un traducteur qui n'a pas cette liberté. La lecture d'une pièce de théâtre moderne peut être indiquée à ce stade puisqu'elle permet de se rendre compte dans quelle mesure la façon de s'exprimer révèle la psychologie d'un personnage. Elle facilitera l'analyse d'un roman où les dialogues jouent un rôle prépondérant.

Ces discussions en classe ne se résument plus à des réactions spontanées comme pendant la première phase. Individuellement ou en mini-groupe, les étudiants préparent " leur " aspect, " leur " personnage ou le résumé d'un article qui traite d'un problème analogue dans la traduction d'une autre œuvre. En général, il est ainsi possible de bien cerner un certain nombre de questions types qui se posent pour le traducteur littéraire. 
Par contre, l'interprétation préalable à une traduction active vise à toucher tous les thèmes et toutes les dimensions du texte et porte autant sur les questions de structure que sur les détails. J'aime mettre à contribution les nouvelles de Hemingway, car il s'agit d'œuvres vraiment très courtes, d'une ou de deux pages, et dont la traduction réussie semble à la portée des étudiants en raison surtout d'un style calqué sur la langue de tous les jours. Ceci permet de démontrer en passant que la littérature n'est pas nécessairement une question de style soutenu et d'images, et que les problèmes auxquels on se heurte malgré les apparences de facilité ne sont qu'accessoirement de nature stylistique.

Pour qu'à ce stade le mode de discussion cadre avec le reste du cours, la lecture ou les lectures successives - et l'interprétation du texte se font d'abord en commun en classe. Chacun fait ensuite sa propre traduction chez lui. Puis, pour réduire à la fois le nombre des traductions à examiner et les erreurs et maladresses qu'elles contiennent, les étudiants forment de petits groupes de trois ou quatre, selon leurs affinités naturelles, et, à partir de leurs brouillons individuels se mettent d'accord sur une traduction commune. Les traductions soumises par les différents groupes sont dactylographiées par la secrétaire et numérotées. La discussion en classe et la justification comparative des quatre ou cinq traductions, obtenues par consensus et de présentation identique, se fait alors sans trop de manifestations de gêne ou d'amour-propre et donne en général de bons résultats.

Comme les lectures et discussions précédentes, la traduction active a surtout une valeur exploratoire. En mettant les étudiants aux prises avec les détails d'un texte, elle leur montre qu'en traduction littéraire aussi il y a souvent plus d'une seule solution; elle leur permet de voir comment des tournures valables en microcontexte peuvent en fait fausser l'impact du texte, et elle les sensibilise et aux possibilités de compensation et à l'effet additif de séries d'écarts à première vue mineurs.

La deuxième partie du cours se termine par des travaux, les étudiants ayant le choix entre le compte rendu d'un article, la comparaison d'articles de différents auteurs sur un même aspect de la traduction littéraire, ou, si l'expérience de la traduction active a été suffisamment concluante, la traduction d'une autre nouvelle de Hemingway. Cette clernière sera auparavant analysée en classe, ce qui permet d'entrevoir en passant l'existence de réseaux de correspondance entre les cuvres d'un même auteur et l'aide que ceux-ci apportent à l'interprétation.

Alors que les travaux à remettre à ce stade sont en fait de véritables épreuves de contrôle, le grand travail individuel de la troisième partie constitue un élément du processus d'apprentissage et prend la forme d'un enseignement individualisé. Il ne s'agit pas de laisser travailler l'étudiant et de juger le résultat à la fin, mais de le guider au cours de son travail en fonction de ses propres capacités et de l'œuvre choisie, et de s'assurer qu'il arrive à des résultats valables qu'il communiquera au groupe. Pendant quelques semaines, le nombre des cours en commun est alors réduit de moitié. Le rythme allégé permet de libérer les étudiants pour leur travail et le professeur pour les rencontres individuelles et de prévoir, à la toute fin du semestre, une cadence de cours plus intense pour la présentation des exposés. En attendant, les étudiants reçoivent des cours de type magistral, qui ne leur demandent aucune préparation, sur les organisations nationales et internationales des traducteurs littéraires, leurs doléances, leurs colloques, les prix littéraires et les prix de traduction, les rapports avec les éditeurs, la convention de Berne et les droits d'auteur, l'importance de la traduction littéraire au Canada, etc.

Après la lecture attentive de "leur» œuvre, les étudiants travaillent selon une grille convenue d'avance et qui sert aussi de trame aux exposés. Elle comporte, dans un premier temps, la biographie sommaire de l'auteur, la description de l'ensemble de son cuvre, la place occupée dans cette œuvre par le roman en question et la présentation 
succincte de celui-ci. Dans un deuxième temps, il s'agit de faire une présentation analogue du traducteur, surtout s'il est lui-même écrivain, en soulignant le cas échéant les affinités apparentes, et d'indiquer s'il a traduit d'autres œuvres et s'il a écrit sur son expérience de traduction ou sur l'auteur qu'il traduit.

Ces recherches représentent souvent un travail considérable - que le professeur peut alléger en guidant l'étudiant d'emblée vers des sources pertinentes -, mais elles habituent les étudiants à voir l'œuvre dans un contexte plus large, et elles leur permettent de se rendre compte des exigences de la traduction littéraire et de mieux juger dans quelle mesure le traducteur semble les avoir respectées.

L'essentiel de l'exposé porte cependant sur l'interprétation de l'œuvre, ou plus exactement, sur la présentation d'un certain nombre de traits caractéristiques que le traducteủr doit respecter, en transposant si nécessaire. Ce sont des questions d'atmosphère, de symbolisme, de leitmotiv bien sûr, mais souvent aussi des éléments formels comme les noms des lieux et des personnages, les citations ou allusions, les tics ou les parlers différenciés des protagonistes, les appellations futuristes, les créations linguistiques, bref, tout ce qui contribue à la particularité de l'œuvre dans sa forme originale. Ils peuvent être traités en énumération ou à partir de quelques scènes clés du roman.

L'interprétation de la traduction reprend ensuite chacun de ces éléments pour examiner comment il se présente dans le nouveau texte et pour justifier, ou le cas échéant critiquer, la solution choisie par le traducteur, qui n'a peut-être pas toujours été aussi cohérent dans ses choix que le texte original le demandait.

Certes, la grille suivie peut paraître trop générale aux traducteurs littéraires, mais elle correspond assez bien aux buts d'un cours de sensibilisation pour les étudiants du premier cycle. Tout en leur laissant une certaine liberté dans le choix des aspects qu'ils désirent traiter plus en détail, elle les éloigne de l'approche de l'explication de texte d'un "passage représentatif » et dirige leur attention sur les éléments caractéristiques tout au long de l'œuvre, et sur leur poids respectif. Les exemples photocopiés fournis par l'étudiant consistent en général en une vingtaine de pages de paragraphes entiers dans lesquels les tournures ou phrases auxquelles l'étudiant se réfere dans son exposé sont soulignées et numérotées. Non seulement les difficultés et les solutions peuvent ainsi être présentées de façon thématique, mais les étudiants du groupe qui ne connaissent pas encore le livre ou sa traduction peuvent se faire une meilleure idée de l'allure des deux écritures.

Il est évident que les exposés, tout en étant bien faits et intéressants, ne constituent pas nécessairement un travail révolutionnaire - bien qu'il y ait parfois des exceptions - et que l'analyse ne dépasse souvent pas ce qui est évident pour un lecteur attentif et averti. Mais ces exposés prouvent en général que les étudiants ont su dégager la structure interne de l'œuvre, qu'ils sont sensibles aux multiples relations et reflets, et qu'ils savent évaluer une traduction par une approche qui correspond à celle des deux cartes mentales dont parle Holmes ${ }^{9}$, une approche pour laquelle la cohérence des choix est un critère primordial. Il est d'ailleurs assez frappant de voir à quel point la nature des écarts permet de détecter le type de traducteur et de distinguer l'écrivain créateur du tâcheron débordé et superficiel.

La portée de chaque analyse individuelle est renforcée ou nuancée par les exposés sur d'autres œuvres, où l'on trouve soit des caractéristiques très différentes, soit des traits analogues et des solutions de traduction différentes, ce qui développe chez les étudiants une certaine résistance à la simplification dogmatique. Pour toutes ces raisons, il est très important de veiller à un minimum de diversification dans le choix des œuvres. L'idéal est d'aboutir à un éventail assez large comportant par exemple une œuvre plus 
ancienne (souvent traduite plus d'une fois), une œuvre canadienne, un roman américain, au moins un auteur féminin (Virginia Woolf traduite par Clara Malraux ou Marguerite Yourcenar), une adaptation par un écrivain, des traducteurs connus et des traducteurs au nom obscur. Si l'on veut obtenir des travaux satisfaisants et pour l'étudiant et pour ceux qui l'écoutent, il faut non seulement assurer une certaine diversité des textes mais encore se limiter à des œuvres littéraires de qualité, marquées par la personnalité créatrice de l'auteur. Les romans à succès et à grande diffusion se prêtent en général très mal à une analyse intéressante et appartiennent plutôt au domaine de la traduction générale. Je ne les exclus cependant pas systématiquement, car si un étudiant averti de ces inconvénients persiste dans le choix d'une telle ouvre, c'est une occasion pour lui et pour le groupe de faire l'expérience de différents niveaux de création et d'écriture, et de leurs conséquences pour la traduction.

BILAN

Les résultats du cours, tels qu'ils se dégagent des exposés, des commentaires spontanés et des évaluations formelles, semblent justifier la démarche suivie. Certes, les étudiants n'auront pas appris à faire de la traduction littéraire, ni à théoriser sur elle, mais ils auront acquis ou développé un sens plus sûr des nuances en prenant conscience des nombreux aspects d'une œuvre qui entrent en ligne dc compte et de leur rôle pour le traducteur, pour le lecteur de la traduction et pour le critique. Ils auront entrevu la réalité matérielle du travail d'un traducteur littéraire, et acquis une vue d'ensemble de ce qui a été écrit à ce sujet par les traducteurs de notre époque qui s'efforcent de rendre justice à l'œuvre. Dans une période où les programmes de traduction sont axés sur le professionnalisme, et négligent en général la culture littéraire de l'étudiant au profit des cours d'introduction à l'économie, aux sciences politiques et aux sciences, ils auront reçu un complément de formation dont l'un des mérites est d'échapper au raisonnement utilitaire. En même temps, le cours entraîne des retombées indéniables au niveau linguistique, grâce à des lectures étendues en langue de départ et d'arrivée, au niveau stylistique, grâce à l'analyse approfondie des deux versions de l'œuvre, et au niveau méthodologique, grâce à l'application répétée de la démarche qui va de l'ensemble au détail et qui, toutes proportions gardées, est également valable en traduction pragmatique.

Le succès du cours est certes en partie attribuable à la formule de cours non obligatoire réunissant un petit nombre d'étudiants, et d'étudiants intéressés. Mais s'ils aiment le cours, c'est aussi parce qu'il leur permet d'échapper aux limites de leur propre savoir-faire et de réagir aux textes non pas comme victimes mais comme consommateurs de plus en plus avertis. L'individualisation du travail, l'expérience d'être le spécialiste d'un auteur ou d'une œuvre en particulier, s'avère de plus un stimulant important et pour leur propre travail et pour celui de leurs camarades. Et l'on peut espérer que le contact avec une matière noble, une production de l'esprit humain qui témoigne à la fois de ses facultés de création et de ses facultés de résistance aux pressions de la réalité, leur aura permis de découvrir une source d'inspiration et de réflexion, qu'ils exploreront davantage pendant leurs loisirs.

Notes

1. Université de Moncton (N.-B.)

2. Cf. Danica Séleskovitch (1984) : "Interpréter un discours n'est pas traduire une langue", in : Séleskovitch/Lederer, Interpréter pour traduire, Paris, Didier, p. 113.

3. Jean Delisle (1980) : l'Analyse du discours comme méthode de traduction, Éditions de l'Université d'Ottawa, p. 85.

4. Sources possibles : META, Babel, congrès de la FIT, actes de colloques, recueils.

5. William Shakespeare : As you like it, Act III, Scene II. 
6. Shakespeare : Comme il vous plaira, acte III, scène II, traduction de François-Victor Hugo (fils de Victor Hugo). De nombreuses éditions, dont : le Théâtre complet de William Shakespeare, Éditions Rencontre, Paris, Garnier, 1961-1964, vol. VIII, p. 93.

7. Shakespeare : Comme il vous plaira, III, 2. Traduction et adaptation de Jean Anouilh et Claude Vincent dans : Shakespeare: Trois comédies, Paris, Gallimard, Livre de poche, 1966, p. 84.

8. Ellipse $\mathrm{n}^{\circ} 16$, la Poésie acadienne / Maritime Poetry.

9. Cf. James S. Holmes (1977) : "Translating Theory, Translation Studies, and the Translator ", in : la Traduction, une profession, Actes du VIII e congrès mondial de la FIT, Montréal, 1977, p. 59 : "I have suggested that actually the translation process is a multi-level process; while we are translating sentences, we have a map of the original text in our minds and at the same time a map of the kind of text we want to produce in the target language. $)$ 